\title{
Insight to Newer Agents and Methods for Local Anesthesia in Pediatric Dentistry
}

\author{
Vijay Prakash Mathur ${ }^{1}$ (D) $\cdot$ Gauri Kalra ${ }^{2}$
}

Received: 29 January 2020 / Accepted: 30 January 2020 / Published online: 8 February 2020

(C) Dr. K C Chaudhuri Foundation 2020

Local anesthetics are being used in various forms for topical use and are used for anesthesia, pain relief and for diagnostic purposes. Many of these salts have similar chemical structures, and somewhat different pharmacokinetic properties and variable pharmacodynamic effects. All these influence the selection of agents for use in various clinical situations.

In the article, titled Comparison of $0.5 \%$ centbucridine and $2 \%$ lignocaine as local anesthetic agents for dental procedures in children: a randomised controlled trial published in current issue of IJP, the authors have addressed the limited application of centbucridine as a local anesthetic agent in pediatric dentistry [1]. The literature is factually scarce in this field even though centbucridine being shown as superior over Lignocaine. The article highlights the superior properties of Centbucridine such as anti-allergic properties and antihistaminic properties, though the results obtained in the clinical trial are not significant [1].

It has been a well-established fact that younger children experience pain and discomfort due to dental infections, injuries and dental procedures [2]. The newer salts available, which are little structurally different to lignocaine, are levobupivacaine, ropivacaine, and articaine [3]. These newer salts are found to be having equal or more efficacy, faster onset of action and longer duration. However, the research is in progress and we may see them as products in the market in coming years. Some of the adjuvants like bicarbonates and steroids have also been tried to improve the efficacy/ duration of action of local anaesthetics in dentistry. The

Vijay Prakash Mathur

vijaymathur7@yahoo.com

1 Division of Pedodontics and Preventive Dentistry, Centre for Dental Education and Research, WHO Collaborating Centre for Oral Health Promotion, All India Institute of Medical Sciences, New Delhi 110029, India

2 Department of Pedodontics and Preventive Dentistry, Sudha Rustogi Dental College, Faridabad, Haryana, India centrbucridine salt has been developed by an academic institute in India and it is expected that it is not likely to be very expensive or maybe equally efficacious.

The clinicians treating children also face a challenge in terms fear of injection or complex instruments. In order to overcome such challenges, newer methods of local anesthesia delivery systems have come into market and it is worthwhile to enumerate them here.

- Computer controlled local anesthesia delivery (CCLAD) system: This advanced system injects local anesthetic agent into the tissues at a set slow speed and also takes into account the anatomical features of the site being injected. Baghlaf et al. and Mittal et al. has reported CCLAD to be advantageous in delivering anesthesia in young patients $[4,5]$.

- Jet Injections: Such system allows the liquid local anesthetic agent to be deposited subcutaneously through the mechanism of mechanical energy though without the use of syringes. It works well in needle phobic young and adult patients. Munshi et al. found a significant acceptance to application of MADAJET XL system over traditional syringes in 3-13 y old children [6].

- Topical Anesthesia: Topical/ local application of local anesthetic agents in gel or cream form have been extensively used in performing dental treatment in pediatric dentistry. Eutectic mixtures for LA (EMLA) have lower melting points and are thoroughly absorbed in the oral mucosa, majorly available as $2.5 \%$ prilocaine and lignocaine in 1:1 ratio. Intranasal sprays as a $3 \%$ tetracaine hydrochloride and $0.05 \%$ oxymetazoline mixture are also available and mainly used to anesthetize upper front teeth.

- LASER Analgesia: Application of LASER is a newer method of reducing pain, though it does not provide complete analgesia but causes alterations in the sodiumpotassium pump, thereby disrupting the impulse conduction to brain temporarily. Soft tissue procedures such as tongue tie removal or treating high frenum attachments 
may be performed with ease without injecting local anesthesia [7].

To conclude, newer local anesthetic delivery systems are the cornerstones of current evidence based pediatric dental practice. Incorporation of such devices not only helps in providing painless dental treatment to young children but also helps in instilling a positive attitude towards dental treatments and oral hygiene.

\section{Compliance with Ethical Standards}

\section{Conflict of Interest None.}

\section{References}

1. Comparison of $0.5 \%$ centbucridine and $2 \%$ lignocaine as local anesthetic agents for dental procedures in children: a randomised controlled trial. Indian J Pediatr. 2020. https://doi.org/10.1007/s12098019-03161-6
2. Pain Management in Infants, Children, Adolescents and Individuals with Special Health Care Needs. Available at: https://www.aapd.org/ globalassets/media/policies_guidelines/ bp_pain.pdf. Accessed 27th December 2019.

3. Shipton EA. New formulations of local anaesthetics- part I. Anesthesiol Res Pract. 2012;2012: Article ID 546409.

4. Baghlaf K, Alamoudi N, Elashiry E, Farsi N, El Derwi DA, Abdullah AM. The pain-related behavior and pain perception associated with computerized anesthesia in pulpotomies of mandibular primary molars: a randomized controlled trial. Quintessence Int. 2015;46:799806.

5. Mittal M, Kumar A, Srivastava D, Sharma P, Sharma S. Pain perception: computerized versus traditional local anesthesia in pediatric patients. J Clin Pediatr Dentistry. 2015;39:470-4.

6. Munshi AK, Hegde A, Bashir N. Clinical evaluation of the efficacy of anesthesia and patient preference using the needle-less jet syringe in pediatric dental practice. J Clin Pediatr Dentistry. 2001;25:131-6.

7. Kulkarni N, Parakh A, Modi S, Mankare A, Vanjari G, Fernandes G. Painless anaesthesia in pediatric dentistry: an updated review. IOSR J Dental Med Sci. 2019;18:67-71.

Publisher's Note Springer Nature remains neutral with regard to jurisdictional claims in published maps and institutional affiliations. 